\title{
Addressing the Liver Progenitor Cell Response and Hepatic Oxidative Stress in Experimental Non- alcoholic Fatty Liver Disease/non-alcoholic Steatohepatitis Using Amniotic Epithelial Cells
}

\author{
Mihiri Goonetilleke \\ Hudson Institute of Medical Research \\ Nathan Kuk \\ Monash University \\ Jeanne Correia \\ Monash University \\ Alex Hodge \\ Monash University \\ Gregory Moore \\ Monash University \\ Michael Gantier \\ Monash University \\ George Yeoh \\ Harry Perkins Institute of Medical Research \\ William Sievert \\ Monash University \\ Rebecca Lim ( $\square$ rebeccca.lim@hudson.org.au ) \\ The Ritchie Centre Hudson Institute of Medical Research Department of Obstetrics and Gynaecology \\ Monash University https://orcid.org/0000-0002-0410-497X
}

\section{Research}

Keywords: Fatty liver disease, NASH/NAFLD, liver progenitor cells, hepatic oxidative stress, amnion epithelial cells, regenerative medicine

Posted Date: November 2nd, 2020

DOl: https://doi.org/10.21203/rs.3.rs-99754/v1

License: (c) (i) This work is licensed under a Creative Commons Attribution 4.0 International License. Read Full License 
Version of Record: A version of this preprint was published at Stem Cell Research \& Therapy on July 28th, 2021. See the published version at https://doi.org/10.1186/s13287-021-02476-6. 


\section{Abstract}

Background: Non-alcoholic steatohepatitis (NASH) is the most common liver disease globally and can progress to cirrhosis and hepatocellular carcinoma (HCC). Currently, patient education and lifestyle changes are the major tools to prevent the continuous progression of NASH. Emerging therapies in NASH target known pathological processes involved in the progression of the disease including inflammation, fibrosis, oxidative stress and apoptosis. Human amniotic epithelial cells (hAECs) were previously shown to be beneficial in experimental models of chronic liver injury, reducing hepatic inflammation and fibrosis. Previous studies have shown that the interaction between liver progenitor cells (LPCs) plays a significant role in the development of fibrosis and HCC in mouse models of fatty liver disease. In this study, we examined the effect hAECs have on the LPC response and hepatic oxidative stress in an experimental model of NASH.

Methods: Experimental NASH was induced in C57BL/6J male mice using a high-fat, high fructose diet for 42 weeks. Mice received either a single intraperitoneal injection of $2 \times 10^{6}$ hAECs at week 34 or an additional hAEC dose at week 38 . Changes to the LPC response and oxidative stress regulators were measured.

Results: hAEC administration significantly reduced the expansion of LPCs and their mitogens, IL-6, IFNY and TWEAK. hAEC administration also reduced neutrophil infiltration and myeloperoxidase production with a concurrent increase in heme oxygenase-1 production. These observations were accompanied by a significant increase in total levels of anti-fibrotic IFNb in mice treated with a single dose of hAECs, which appeared to be independent of c-GAS-STING activation.

Conclusions: Expansion of liver progenitor cells, hepatic inflammation and oxidative stress associated with experimental NASH were attenuated by hAEC administration. Given that repeated doses did not significantly increase efficacy, future studies assessing the impact of dose escalation and/or timing of dose may provide insights into clinical translation.

\section{Background}

Non-alcoholic Fatty Liver Disease (NAFLD) is the most common liver disease globally [1]. While global prevalence of NAFLD varies widely, the incidence rates in some countries are as high as $45 \%$ [2]. As many as one-third of those affected by NAFLD may progress to inflammation-associated fibrotic disease and cirrhosis (non-alcoholic steatohepatitis; NASH) [3] and eventually hepatocellular carcinoma (HCC). The global prevalence of NAFLD is currently estimated at 25\% [4], and in 2020, NASH replaced hepatitis C as the leading reason for liver transplantation. While patient education and lifestyle changes are considered the major tools to prevent progression of liver disease, there is currently no cure for NAFLD/NASH. With the failure of several drugs in late stage clinical development and for patients who are not transplantable, alternative options must be explored. 
Emerging therapies in NASH target known pathological processes and pathways involved in the progression of the disease including inflammation, fibrosis, oxidative stress and apoptosis. Given previous reports suggesting that liver progenitor cells (LPCs) are cellular targets for malignant transformation in hepatocellular carcinoma (HCC), and the increased risk of HCC in NALFD/NASH [5-9], LPCs have been identified as a possible target for treatment [10]). Furthermore, anti-oxidants, including NAcetylcysteine (NAC) and S-adenosylmethionine (SAMe), have been explored as potential treatment for NASH [11-13] since oxidative stress has been implicated in the progression of the LPC response [14] and pathogenesis of NASH. Recent pre-clinical studies have also explored interferon b (IFNb) as a potential anti-fibrotic for $\mathrm{NASH}$, with its ability to downregulate fibrogenic genes associated with TGFb-1 and MyD88 pathways [15]. While these emerging treatments have shown varying degrees of success, there remains an urgent need to develop efficacious therapies that address the complex pathophysiologic processes implicated in NASH.

Cell-based therapies have shown promising results in the treatment and prevention of experimental NAFLD/NASH $[16,17]$. Human amnion epithelial cells (hAEC), that line the amniotic sac of the placenta are non-tumorigenic and immunologically privileged. The therapeutic potential of hAECs has been explored in the setting of liver [18], lung [19], cardiac [20], epidermal [21] and neurological injury [22]. We $[16,18,23-26]$ and others $[27,28]$ postulate that hAECs may be a promising alternative to address NAFLD/NASH either through the restriction of hepatocyte death and stellate cell activation [24] and/or through the modification of dominant macrophage phenotype[18, 24]. Both hAECs and their secretome have been shown to reduce chronic liver injury $[23,24]$. While the anti-inflammatory and anti-fibrotic effects of hAECs have been extensively explored in carbon tetrachloride models of liver injury [18, 23-25], their effect on liver regeneration in the context of NAFLD/NASH, is poorly understood.

In this study, we investigate the influence hAECs have on the LPC response and hepatic oxidative stress, in a murine model of human NASH. Previous reports show that hAECs dampen hepatic inflammation and fibrosis in experimental NASH [24], but the mechanisms through which hAECs, and other cell-based modalities, influence the LPC response and oxidative stress response remain unknown.

\section{Methods}

\section{Ethics statement}

This study was approved by Monash University Animal Ethics Committee (AE\# B13/01) and conducted in accordance with the Australian Code of Practice for the Care and Use of Animals for Scientific Purposes (2006). All mice were monitored daily. The Monash Health Human Research Ethics Committee approved the collection and use of human amnion (Monash Health HREC approval numbers: 01067B, 12223B). Informed written consent was obtained from each patient prior to surgery.

\section{Isolation of hAECs}


The hAECs were isolated as previously described [29]. Briefly, amniotic membranes were separated from underlying chorions, washed in Hanks Balanced Salt Solution (HBSS) and digested in $0.05 \%$ trypsin-EDTA (cat no. 25300062 , Thermo Fisher Scientific, Waltham, MA) for one hour at $37^{\circ} \mathrm{C}$. Only batches with $>90 \%$ cellular viability were cryopreserved in liquid nitrogen for use in this study. hAECs isolated from 4 donors were used for this animal study.

\section{Animals and Experimental Schedule}

Seven-week-old male C57BL/6J mice were purchased from Monash Animal Services (Monash University, Melbourne, Australia). Mice were divided into four groups ( $n=6-8)$, one group receiving ad libitum standard chow with normal water (Normal), and the other three groups receiving a modified experimental NASH diet with high fructose corn syrup (Table 1). The three groups receiving the experimental NASH diet were either on the diet alone (FF), received a single dose of hAECs (FFHS) or received two doses of hAECs (FFHD) (Figure 1). Control mice included standard chow fed and mice on the experimental NASH diet alone (FF). All mice were culled at Week 42.

\section{Immunohistochemistry and immunofluorescence}

Paraffin-embedded liver sections from all treatment groups were dewaxed, rehydrated, and incubated in $10 \mathrm{mM}$ sodium citrate $\mathrm{pH} 6$ or $10 \mathrm{mM}$ Tris-EDTA pH 9 for heat mediated antigen retrieval (Table 2). Endogenous peroxidase activity was blocked using $3 \% \mathrm{H}_{2} \mathrm{O}_{2}$. Tissue sections were blocked with a universal protein blocking solution for $1 \mathrm{~h}$ and then incubated with primary antibodies overnight at $4^{\circ} \mathrm{C}$ as per Table 2. Tissue sections were then washed three times and incubated with secondary antibodies (Table 2) for 1 hour. For PanCK, antibody binding was detected using Vectastain ABC HRP kit (Vector Laboratories, Meadowbrook, QLD, Australia) followed by DAB chromogen (Dako, Mulgrave, Victoria, Australia). Sections for MPO, CD45, NIMP-R14, and HO-1 were incubated with DAPI (Sigma-Aldrich, St. Louis, MO, United States) for 10 mins. Data are presented as number of cells per field of view (PanCK) or percentage (\%) positive cells per five non-overlapping fields at 20x magnification (CD45, NIMP-R14, MPO and $\mathrm{HO}-1$ ) normalised to the number of DAPI positive cells using Image J (v1.53c, National Institutes of Health, USA).

\section{RNA isolation and RT-PCR}

Total RNA was isolated from mouse liver tissue or cultured cells using the RNeasy mini-kit according to the manufacturer's instructions (Qiagen Pty Ltd, Hilden, Germany). cDNA was synthesised using the HighCapacity Reverse Transcription Kit (Applied Biosystems, CA, USA) and amplified using Power SYBR ${ }^{\text {TM }}$ Green PCR Master Mix (Applied Biosystems, CA, USA) for qPCR. Quantitative RT-PCR (QuantStudio Realtime PCR system) was used to examine gene expression levels of Nox2, Nox4, Sting, II-6, Tweak, Ifny, Ifn $\beta$, Rsad2, Ifit1, Ifih1 and Isg15 (Table 3). Data were normalized to the housekeeping gene $18 \mathrm{~s}$ with fold change calculated using delta cycle-threshold method [30]. Melting curves were used in each run to confirm specificity of amplification. 
The iMACs (immortalised mouse macrophages) and BMOLs (bipotential murine oval liver cells), a mouse LPC cell line, were co-cultured with hAECs from 4 donors in 6-well plates in $0.4 \mathrm{~mm}$ transwells at a 1:1 ratio. Wells containing only iMACs or BMOLs served as negative controls, maintained in DMEM:F12 supplemented with 5-10\% FBS or DMEM:F12 supplemented with $30 \mathrm{ng} / \mathrm{mL}$ IGF-II, $50 \mathrm{ng} / \mathrm{mL}$ EGF, 10 $\mu \mathrm{g} / \mathrm{mL}$ insulin, $100 \mathrm{U} / \mathrm{mL}$ penicillin and streptomycin and 5-10\% FBS, respectively. Cells treated with 5,6Dimethylxanthenone-4-acetic Acid (DMXAA-D5817, Sigma-Aldrich, St. Louis, MO, United States) served as positive controls for CGAS-STING activation. Cultures were maintained at $37^{\circ} \mathrm{C}$ in $95 \%$ humidity and $5 \%$ $\mathrm{CO}_{2}$ for 2 hours prior to RNA isolation.

\section{Data Analysis}

Data were analyzed using GraphPad Prism version 6.0 software (GraphPad Software, San Diego, CA, United States). Multiple comparisons between different groups were analyzed by one-way ANOVA. Differences were considered statistically significant when $p<0.05$; and calculations and graphs are presented as mean \pm standard error of mean.

\section{Results}

\section{hAECs reduced LPC response in experimental non-alcoholic steatohepatitis}

The experimental NASH diet significantly increased (5.5-fold) the number of LPCs (PanCK +cells) in the liver (Normal vs FF; $21.47 \pm 0.18$ vs. $117.8 \pm 11.5$, Figure $2, p<0.0001$ ). A single dose of hAECs significantly decreased the number of LPCs (FF vs FFHS; $117.8 \pm 11.5$ vs. $76.27 \pm 5.9$, Figure $2, p=0.005$ ), but no significant reduction was seen in the group that received a second dose of hAECs. It is worth noting that hAEC administration did not return LPC numbers to control levels. The number of PanCK+ cells in the FFHS and FFHD groups remained elevated compared to healthy controls (Normal vs FFHS; $21.47 \pm 0.18$ vs. $76.27 \pm 5.9$, Figure $2, p=0.0088$; Normal vs FFHD; $21.47 \pm 0.18$ vs. $91.07 \pm 8.07$, Figure $2, p=0.0019$ ).

Next, we assessed changes to transcriptional levels of known LPC mitogens in whole mouse liver tissue. Here we observed that compared with FF mice, a single dose of hAEC reduced transcription of II- 6 by 20fold (FFHS vs FF; $0.06 \pm 0.03$ vs $1.2 \pm 0.27$, Figure $3 a, p=0.001$ ) and by 24 -fold in the FFHD group (FFHD vs FF; $0.05 \pm 0.029$ vs $1.2 \pm 0.27$, Figure $3 a, p=0.002$ ). The transcription of Tweak was reduced by 7 -fold in the FFHS group $(0.17 \pm 0.06$ vs $1.2 \pm 0.24$, Figure $3 b, p=0.001)$ and was 5 -fold lower in the FFHD group $(0.22 \pm 0.9$ vs $1.2 \pm 0.24$, Figure $3 b, p=0.002)$ compared to the fast food only group. Furthermore, transcriptional levels of Ifny were 23 -fold lower in the FFHS group $(0.04 \pm 0.009$ vs $0.94 \pm 0.2$, Figure $3 c$, $p=0.0003)$ and 15 -fold lower in the FFHD group $(0.06 \pm 0.02$ vs $0.94 \pm 0.2$, Figure $3 c, p=0.0007)$ compared to the FF group.

\section{hAECs reduced neutrophil infiltration and hepatic oxidative stress}


Given previous reports on the role of neutrophil infiltration [31] and central role of oxidative stress in $\mathrm{NASH}$, we next evaluated the effect of hAECs on these aspects of liver pathology. We observed a significant increase in leukocytes (CD45+ cells) in the experimental model of NASH (FF vs Normal; $37.9 \pm 2.9 \%$ vs $21.3 \pm 1.08 \%$, Figure $4, p<0.0001$ ). Furthermore, significantly lower levels of CD $45+$ cells were observed in the FFHS group $(23.25 \pm 1.03 \%$ vs $21.3 \pm 1.08 \%$, Figure $4, p<0.0001)$ and the FFHD group $(29.61 \% \pm 1.7$ vs $21.3 \pm 1.08 \%$, Figure $4, p=0.0076)$ compared to the FF group. Notably, numbers of CD45+ cells were significantly lower in the FFHS compared to the FFHD group $(23.25 \pm 1.03 \%$ vs $29.61 \% \pm 1.7$, Figure 4, $p=0.001$ ). Further, we noted a significant increase in NIMP-R14+ neutrophils in the FF group compared to the control group $(17.8 \pm 1.7 \%$ vs $1.2 \pm 0.2 \%$, Figure $5, p<0.0001)$. Compared to the FF group, the number of NIMP-R14+ neutrophils was significantly reduced in both the FFHS $(10.81 \pm 0.8 \%$ vs $17.8 \pm 1.7 \%, p=0.0001)$ and FFHD group $(9.3 \pm 0.9 \%$ vs $17.8 \pm 1.7 \%$, Figure $5, p<0.0001)$. This coincided with changes in hepatic MPO levels, where we observed a significant increase in the number of MPO+ cells compared to control $(62.7 \pm 7.7 \%$ vs $10.3 \pm 1.8 \%$, Figure $6, p<0.0001)$. Further, the number of MPO+ cells were significantly reduced in FFHS group $(29.3 \pm 3.5 \%$ vs $62.7 \pm 7.7 \%$, Figure $6, p<0.0001)$ and FFHD $(23.7 \pm 3.08 \%$ vs $62.7 \pm 7.7 \%$, Figure $6, p<0.0001)$.

We further investigated the effect of hAECs on oxidative stress by assessing the relative expression of Nox4 and Nox2. We observed a 6-fold reduction in the expression levels of NOX2 in the FFHS group $(0.2 \pm 0.1$ vs $1.2 \pm 0.22$, Figure $7 a p=0.003)$ and a 4.8 -fold reduction in the FFHD group $(0.25 \pm 0.13$ vs $1.2 \pm 0.22$, Figure $7 a, p=0.005$ ). Further, transcriptional levels of NOX4 were lower in hAEC treated mice but this was not statistically significant (Figure $7 b, p=0.10$ ). We next assessed HO-1 production and found that, compared to the FF only group, there was a significant increase in expression of $\mathrm{HO}-1$ in mice treated with a single dose of hAECs $(20.72 \pm 2.1$ vs $30.8 \pm 1.9 \%$, Figure $8, p<0.0001)$ but not a double dose of hAECs. Notably, there was no significant difference in HO-1+ cells between the FFHD and FF group.

\section{hAECs increased total levels of hepatic IFNb, independent of c-GAS-STING activation}

Hepatic levels of IFNb were increased by 4 -fold in the FFHS group compared to the FF group $(1.3 \pm 0.34$ vs $5.6 \pm 1.8$, Figure $9 a, p=0.005)$. Interestingly, this was not observed in the FFHD mice, with a significant difference in the hepatic levels of IFNb between the FFHS and FFHD groups ( $5.5 \pm 1.8$ vs $1.9 \pm 1.3$, Figure $9 a, p=0.03)$. No significant differences were observed in the expression of several IFN-induced genes (Rsad2, Ifit1, Isg15 and Ifih1) between treatment groups (data not shown). Furthermore, the expression of STING in the FFHD group was 10 -fold higher than the FF group ( $36.6 \pm 8.7$ vs $3.5 \pm 0.8$, Figure $9 b$, $p<0.0001)$. In order to investigate whether the increase in hepatic IFN- $\beta$ in the FFHS group was due to a direct effect on c-GAS-STING, we performed a series of in vitro studies, co-culturing hAECs with iMACs or BMOLs. Here we observed that co-culturing with hAECs did not significantly alter the expression of IFIT1 (Figure 10a), IFN $\beta$ (Figure 10b) and RSAD2 (Figure 10c) in iMACs. Similarly, co-culturing with hAECs did not significantly alter the expression of IFIT1 (Figure 10d), IFN 3 (Figure 10e) and RSAD2 (Figure 10f) in BMOLs. These findings suggest that the increase in hepatic IFN- $\beta$ expression in the FFHS group was unlikely to be due to a direct effect of hAECs on iMACs or BMOLs - the major responders to cGAS-STING signalling in the liver. 


\section{Discussion}

The main objective of this study was to determine the impact of hAECs on the LPC population and hepatic oxidative stress, in a murine model of human NASH. Previous reports show that hAECs dampen hepatic inflammation and fibrosis in experimental NASH [24], but the mechanisms through which hAECs, and other cell-based modalities influence the LPC response remain unknown. In this study, we demonstrate that hAECs dampen the LPC response in experimental NASH through the reduction of critical LPC mitogens including TWEAK, IL-6 and IFNg. Interestingly, we also observed that hAEC administration reduced leucocyte and specifically neutrophil infiltration and myeloperoxidase production with concurrent increase in $\mathrm{HO}-1$ production. This observation was accompanied by an increase in total levels of anti-fibrotic IFNb which appeared to be independent of c-GAS-STING activation.

Liver progenitor cells are facultative stem cells that reside in the Canals of Hering. This niche is activated during chronic liver injury when hepatocyte proliferation is insufficient to achieve homeostasis. In a fibrogenic environment where TGFb levels are persistently elevated, the balance is tipped towards LPC expansion as hepatocytes undergo TGFb -induced apoptosis [32,33]. The expansion of LPCs is mediated by mitogens that significantly overlap with pro-inflammatory cytokines released by hepatic macrophages, including tumor necrosis factor (TNF), lymphotoxin $\beta$ (LT $\beta$ ), interferon $y$ (IFNY), interleukin 6 (IL-6) and tumor necrosis factor-like weak inducer of apoptosis (TWEAK) $[5,7,26,34-37]$. The reduction in both LPC numbers and expression levels of the LPC mitogens IL-6, IFNY and TWEAK in hAEC treated mice was most likely related to a concomitant reduction in hepatic macrophages. While hAECs increased LPC proliferation in in vitro co-culture studies [26], the reduction in LPC response was most likely achieved by hAEC reduction of hepatic macrophages and their secreted LPC mitogens. We have observed this consistently in previous studies using in vivo liver and lung injury models, that have demonstrated that hAECs reduce macrophage recruitment and support a predominant alternatively activated (M2) phenotype $[18,19]$. It should be noted that while we observed a potent anti-inflammatory effect of hAECs through the reduction in the LPC mitogens, we saw a lesser effect on the LPC numbers. This indicates that other factors including Wnt ligands recently reported to be produced by hAECs[38], may still persist to drive the LPC response in liver homeostasis and repair [39].

We next sought to assess the impact of hAEC treatment on neutrophil infiltration and activation given the relevance of neutrophils and oxidative stress in both adults with NASH and obese children at risk of developing NASH [40, 41]. Indeed, pharmaceutical approaches have been employed to address this contributor to NASH progression [42]. The degranulation of neutrophils releases myeloperoxidase (MPO), a ROS-producing enzyme that oxidises phosphatidylcholine, creating a positive feedback loop by activating more neutrophils while also acting as a ligand for scavenger receptors, thus exacerbating fibrogenesis [43]. Here, we observed that the administration of either a single or double dose of hAECs significantly reduced neutrophil infiltration as determined by NIMP-R14 (Ly-6G/-6C) staining which coincided with a reduction in hepatic MPO levels. Further to this, we observed a significant reduction in the expression levels of NADPH oxidase 2 (NOX2) in mice administered with hAECs at both doses, but no significant difference in NOX4 expression levels. NOXs are a family of enzymes known to produce ROS 
during liver injury [44] and have been implicated in the activation of quiescent HSCs to myofibroblasts [45]. The NOX isoforms are differentially expressed by resident liver cells. Hepatic macrophages only express NOX2, while hepatocytes and hepatic stellate cells express both NOX2 and NOX4 as well as other isoforms [45]. The reduction in NOX2 expression levels following hAEC administration may be attributed to reduced macrophage recruitment previously reported $[19,24,46]$. In addition to the reduction in ROSproducing enzymes, hAEC administration also resulted in an increase in the anti-oxidant HO-1, thus suggesting that the improvements in NASH activity scores, reported in our previous work [24], may have been achieved through a combination of reduced oxidative stress and improved anti-oxidant capacity. Considering the role of ROS in mediating HSC activation, hepatocyte apoptosis, inhibition of hepatocyte replication and accumulation of LPCs $[14,45]$, these findings are extremely encouraging for the clinical translation of hAEC as a therapy for NASH. The ability of hAECs to reduce oxidative stress that enhances oncogenic mutational events in liver cells; in particular proliferating LPCs, is important as it has been shown that attenuation of the inflammatory response reduces the incidence of HCC in mouse models of fatty liver disease $[47,48]$.

The severity of hepatic inflammation has been identified as an independent risk factor for fibrosis progression in NASH [49]. Interferon b (IFNb) was suggested as a potential anti-fibrotic for NASH with its ability to downregulate fibrogenic genes associated with TGFb-1 and MyD88 pathways [15]. As such, we measured total gene expression of IFNb in the livers of NASH mice, whereupon we observed that a single dose of hAECs significantly increased IFNb where a double dose of hAECs failed to achieve this. Given the implication of the c-GAS-STING pathway in the initiation of IFNb expression and progression of liver fibrosis [50,51], we then assessed the gene transcription of STING and IFN-inducible genes in total liver lysates. Here, we observed that STING expression was only significantly increased in the livers of mice exposed to a double dose of hAECs. Furthermore, we did not observe significant expression of common IFN-induced genes. Next, we performed in vitro studies using immortalised macrophages (iMACs) and a mouse LPC cell line (BMOLs). No changes in the expression of Ifnb, Ifit1 and Rsad2 genes in iMACs or BMOLs following co-culture with hAECs were observed. hAECs did not lead to IFN induction in vitro and did not result in the initiation of c-GAS-STING signalling. Together, these data do not support a role for hAECs in cGAS-STING activation. Additionally, increased hepatic STING transcription while coincident with the double dose of hAECs does not appear to be a direct consequence of hAEC administration. It is important to recognise that the co-culture studies were limited by use of immortalised macrophages and BMOLs. Future co-culture studies using primary cells or transcriptional profiling of flow sorted cell populations may provide further insights into the cell-cell interactions. CGAS or STING knockout cell lines and/or mouse strains would be beneficial to ascertain the impact of hAECs on the cGAS-STING pathway. This is particularly important given the growing evidence that the DNA-sensing cGAS-STING pathway is critical to NASH progression [50].

\section{Conclusion}

In summary, we provide the first evidence that hAECs reduced the LPC response in experimental NASH. This was likely achieved through a reduction in LPC mitogens as shown in this study, and possibly 
mediated by the reduction in hepatic macrophages observed in previous work [24]. We also report for the first time that hAEC administration reduced inflammation and hepatic levels of ROS-producing enzymes concomitant with increased anti-oxidant capacity. We observed an increase in antifibrotic IFNb following hAEC administration that appeared to be independent of CGAS-STING pathway activation. These findings are supportive of clinical translation of hAECs as a therapy for NASH. The differences seen in this study between single versus double doses of hAECs suggests that dose escalation trials should be designed to assess optimal dose rather than maximal tolerable dose. Future studies should also assess the efficacy of repeated doses compared to increased doses.

\section{List Of Abbreviations}




\begin{tabular}{|ll|}
\hline BMOL & Bipotential Murine Oval Liver \\
\hline DMXAA & 5,6-Dimethylxanthenone-4-acetic Acid \\
\hline FF & Fast Food diet \\
\hline hAEC & Human Amniotic Epithelial Cells \\
\hline HBSS & Hanks Balanced Salt Solution \\
\hline HCC & Hepatocellular Carcinoma \\
\hline HO-1 & Heme Oxygenase 1 \\
\hline HSC & Hepatic Stellate Cells \\
\hline IF & Immunofluorescence \\
\hline IFNb & Interferon b \\
\hline IFNY & Interferon Y \\
\hline IHC & Immunohistochemistry \\
\hline IL-6 & Interleukin 6 \\
\hline iMAC & Immortalised Mouse Macrophages \\
\hline IP & Intraperitoneal \\
\hline LPC & Liver Progenitor Cell \\
\hline LT $\beta$ & Lymphotoxin $\beta$ \\
\hline MPO & Myeloperoxidase \\
\hline NAC & N-Acetylcysteine \\
\hline NAFLD & Non-Alcoholic Fatty Liver Disease \\
\hline NASH & Non-Alcoholic Steatohepatitis \\
\hline NOX & NADPH oxidase \\
\hline ROS & Reactive Oxygen Species \\
\hline RT-PCR & Real time quantitative polymerase chainreaction \\
\hline SAMe & S-adenosylmethionine \\
\hline TWEAK & Tumor Necrosis factor-like Weak Inducer of Apoptosis Error of Mean \\
\hline
\end{tabular}




\section{Declarations}

\section{Ethics approval and consent to participate}

This animal study was approved by Monash University Animal Ethics Committee (AE\# B13/01) and conducted in accordance with the Australian Code of Practice for the Care and Use of Animals for Scientific Purposes (2006). The Monash Health Human Research Ethics Committee approved the collection and use of human amnion (Monash Health HREC approval numbers: 01067B, 12223B). Informed written consent was obtained from each patient prior to surgery.

\section{Consent for publication}

Not applicable

\section{Availability of data and materials}

The datasets used and analysed during the current study are available from the corresponding author on request.

\section{Competing interests}

The authors declare that they have no competing interests

\section{Funding}

This work was supported by an MRFF Stem Cell Mission grant (APP1201805).

\section{Authors' contributions}

MG, NK, JC, AH performed the experiments. MG and RL analyzed the data. RL and MPG conceived and designed all experiments. MG and RL wrote the manuscript. RL, GM and WS were responsible for the grant application. RL, GM, GY and WS edited the manuscript. All authors have read and approved the final manuscript.

\section{Acknowledgements}

The authors thank Associate Professor Ashley Mansell (Hudson Institute of Medical Research, Australia) for his kind gift of iMAC cells. The authors acknowledge the facilities and scientific and technical assistance of the Monash Health Translation Precinct (MHTP) Medical Genomics Facility, the MHTP Monash Micro Imaging Platform, the MHTP Monash Animal Research Platform (MARP) and the Monash Histology Platform, Department of Anatomy and Developmental Biology, Monash University.

\section{References}


1. Cotter, T.G. and M. Rinella, Nonalcoholic Fatty Liver Disease 2020: The State of the Disease. Gastroenterology (New York, N.Y. 1943), 2020. 158(7): p. 1851-1864.

2. Torres, D.M., C.D. Williams, and S.A. Harrison, Features, Diagnosis, and Treatment of Nonalcoholic Fatty Liver Disease. Clinical gastroenterology and hepatology, 2012. 10(8): p. 837-858.

3. Singh, S., et al., Fibrosis Progression in Nonalcoholic Fatty Liver vs Nonalcoholic Steatohepatitis: A Systematic Review and Meta-analysis of Paired-Biopsy Studies. Clinical gastroenterology and hepatology, 2015. 13(4): p. 643-654.e9.

4. Younossi, Z.M., et al., Global epidemiology of nonalcoholic fatty liver disease-Meta-analytic assessment of prevalence, incidence, and outcomes. Hepatology, 2016. 64(1): p. 73-84.

5. Knight, B., et al., Interferon- $y$ exacerbates liver damage, the hepatic progenitor cell response and fibrosis in a mouse model of chronic liver injury. Journal of hepatology, 2007. 47(6): p. 826-833.

6. Lowes, K.N., et al., Oval cell numbers in human chronic liver diseases are directly related to disease severity. Am J Pathol, 1999. 154(2): p. 537-41.

7. Nguyen, L.N., et al., Transforming growth factor-beta differentially regulates oval cell and hepatocyte proliferation. Hepatology, 2007. 45(1): p. 31-41.

8. Yeoh, G.C., et al., Opposing roles of gp130-mediated STAT-3 and ERK-1/ 2 signaling in liver progenitor cell migration and proliferation. Hepatology, 2007. 45(2): p. 486-94.

9. Best, J., et al., Role of liver progenitors in liver regeneration. Hepatobiliary Surg Nutr, 2015. 4(1): p. 4858.

10. Marquardt, J.U., Deconvolution of the cellular origin in hepatocellular carcinoma: Hepatocytes take the center stage. Hepatology, 2016. 64(4): p. 1020-1023.

11. Emel Pamuk, G. and A. Sonsuz, N-Acetylcysteine in The Treatment Of Non-Alcoholic Steatohepatitis. Journal of Gastroenterology and Hepatology, 2003. 18(10): p. 1220-1221.

12. Gulbahar, O., et al., Treatment of non-alcoholic steatohepatitis with N-acetyl cystein. Gastroenterology (New York, N.Y. 1943), 2000. 118(4): p. A1444-A1444.

13. Oz, H.S., et al., Glutathione-enhancing agents protect against steatohepatitis in a dietary model. Journal of biochemical and molecular toxicology, 2006. 20(1): p. 39.

14. Roskams, T., et al., Oxidative Stress and Oval Cell Accumulation in Mice and Humans with Alcoholic and Nonalcoholic Fatty Liver Disease. The American Journal of Pathology, 2003. 163(4): p. 13011311.

15. Shimozono, R., et al., Interferon- $\beta$ Mediates Signaling Pathways Uniquely Regulated in Hepatic Stellate Cells and Attenuates the Progression of Hepatic Fibrosis in a Dietary Mouse Model. J Interferon Cytokine Res, 2015. 35(6): p. 464-73.

16. Lim, R., et al., A Pilot Study Evaluating the Safety of Intravenously Administered Human Amnion Epithelial Cells for the Treatment of Hepatic Fibrosis. Frontiers in pharmacology, 2017. 8: p. 549.

17. Lim, R., et al., First-In-Human Administration of Allogeneic Amnion Cells in Premature Infants With Bronchopulmonary Dysplasia: A Safety Study. STEM CELLS Translational Medicine, 2018. 7(9): p. 
628-635.

18. Manuelpillai, U., et al., Human Amniotic Epithelial Cell Transplantation Induces Markers of Alternative Macrophage Activation and Reduces Established Hepatic Fibrosis. PLoS One, 2012. 7(6): p. e38631.

19. Tan, J.L., et al., Human Amnion Epithelial Cells Mediate Lung Repair by Directly Modulating Macrophage Recruitment and Polarization. Cell Transplantation, 2014. 23(3): p. 319-328.

20. Song, Y.-S., et al., Transplanted Human Amniotic Epithelial Cells Secrete Paracrine Proangiogenic Cytokines in Rat Model of Myocardial Infarctio. Cell Transplantation, 2015. 24(10): p. 2055-2064.

21. Zhao, B., et al., Human amniotic epithelial cells attenuate TGF- $\beta 1$-induced human dermal fibroblast transformation to myofibroblasts via TGF- $\beta 1 /$ Smad3 pathway. Cytotherapy, 2016. 18(8): p. 10121024.

22. Leaw, B., et al., Human amnion epithelial cells rescue cell death via immunomodulation of microglia in a mouse model of perinatal brain injury. Stem Cell Research \& Therapy, 2017. 8.

23. Alhomrani, M., et al., The Human Amnion Epithelial Cell Secretome Decreases Hepatic Fibrosis in Mice with Chronic Liver Fibrosis. Frontiers in Pharmacology, 2017. 8.

24. Kuk, N., et al., Human amnion epithelial cells and their soluble factors reduce liver fibrosis in murine non-alcoholic steatohepatitis. Journal of Gastroenterology and Hepatology, 2019. 34(8): p. 14411449.

25. Manuelpillai, U., et al., Transplantation of human amnion epithelial cells reduces hepatic fibrosis in immunocompetent CCID-treated mice. Cell Transplant, 2010. 19(9): p. 1157-68.

26. Hodge, A., et al., Human Amnion Epithelial Cells Produce Soluble Factors that Enhance Liver Repair by Reducing Fibrosis While Maintaining Regeneration in a Model of Chronic Liver Injury. Cell Transplant, 2020. 29: p. 963689720950221.

27. Sant'Anna, L.B., et al., Amniotic membrane application reduces liver fibrosis in a bile duct ligation rat model. Cell Transplant, 2011. 20(3): p. 441-53.

28. Andrewartha, N. and G. Yeoh, Human Amnion Epithelial Cell Therapy for Chronic Liver Disease. Stem Cells Int, 2019. 2019: p. 8106482.

29. Miki, T., et al., Isolation of amniotic epithelial stem cells. Curr Protoc Stem Cell Biol, 2010. Chapter 1: p. Unit 1E.3.

30. Livak, K.J. and T.D. Schmittgen, Analysis of relative gene expression data using real-time quantitative PCR and the 2(-Delta Delta C(T)) Method. Methods, 2001. 25(4): p. 402-8.

31. Bartneck, M. and J. Wang, Therapeutic Targeting of Neutrophil Granulocytes in Inflammatory Liver Disease. Frontiers in immunology, 2019. 10: p. 2257.

32. Gressner, A.M., et al., TGF-beta-mediated hepatocellular apoptosis by rat and human hepatoma cells and primary rat hepatocytes. J Hepatol, 1997. 26(5): p. 1079-92.

33. Oberhammer, F., et al., Effect of transforming growth factor beta on cell death of cultured rat hepatocytes. Cancer Res, 1991. 51(9): p. 2478-85. 
34. Dwyer, B.J., et al., TWEAK and LT $\beta$ Signaling during Chronic Liver Disease. Frontiers in immunology, 2014. 5: p. 39-39.

35. Akhurst, B., et al., Differential lymphotoxin-beta and interferon gamma signaling during mouse liver regeneration induced by chronic and acute injury. Hepatology (Baltimore, Md.), 2005. 41(2): p. 327.

36. Knight, B. and G. Yeoh, TNF/LTa double knockout mice display abnormal inflammatory and regenerative responses to acute and chronic liver injury. Cell and Tissue Research, 2005. 319(1): p. 61-70.

37. Knight, B., et al., Impaired preneoplastic changes and liver tumor formation in tumor necrosis factor receptor type 1 knockout mice. The Journal of experimental medicine, 2000. 192(12): p. 1809.

38. Zhu, D., et al., Prematurity negatively affects regenerative properties of human amniotic epithelial cells in the context of lung repair. Clinical Science, 2020. 134(20): p. 2665-2679.

39. Russell, J.O. and S.P. Monga, Wnt/ $\beta$-Catenin Signaling in Liver Development, Homeostasis, and Pathobiology. Annu Rev Pathol, 2018. 13: p. 351-378.

40. Strauss, R.S., Comparison of serum concentrations of alpha-tocopherol and beta-carotene in a crosssectional sample of obese and nonobese children (NHANES III). National Health and Nutrition Examination Survey. J Pediatr, 1999. 134(2): p. 160-5.

41. Inzaugarat, M.E., et al., Altered phenotype and functionality of circulating immune cells characterize adult patients with nonalcoholic steatohepatitis. J Clin Immunol, 2011. 31(6): p. 1120-30.

42. Bartneck, M. and J. Wang, Therapeutic Targeting of Neutrophil Granulocytes in Inflammatory Liver Disease. Frontiers in immunology, 2019. 10: p. 2257-2257.

43. Ikura, Y., et al., Localization of oxidized phosphatidylcholine in nonalcoholic fatty liver disease: impact on disease progression. Hepatology, 2006. 43(3): p. 506-14.

44. Paik, Y.-H., et al., Role of NADPH oxidases in liver fibrosis. Antioxidants \& redox signaling, 2014. 20(17): p. 2854-2872.

45. Liang, S., T. Kisseleva, and D.A. Brenner, The Role of NADPH Oxidases (NOXs) in Liver Fibrosis and the Activation of Myofibroblasts. Frontiers in physiology, 2016. 7: p. 17-17.

46. Hodge, A., et al., Soluble factors derived from human amniotic epithelial cells suppress collagen production in human hepatic stellate cells. Cytotherapy (Oxford, England), 2014. 16(8): p. 1132-1144.

47. Passman, A.M., et al., A modified choline-deficient, ethionine-supplemented diet reduces morbidity and retains a liver progenitor cell response in mice. Dis Model Mech, 2015. 8(12): p. 1635-41.

48. Viebahn, C.S., et al., Invading macrophages play a major role in the liver progenitor cell response to chronic liver injury. J Hepatol, 2010. 53(3): p. 500-7.

49. Argo, C.K., et al., Systematic review of risk factors for fibrosis progression in non-alcoholic steatohepatitis. J Hepatol, 2009. 51(2): p. 371-9.

50. Luo, X., et al., Expression of STING Is Increased in Liver Tissues From Patients With NAFLD and Promotes Macrophage-Mediated Hepatic Inflammation and Fibrosis in Mice. Gastroenterology (New York, N.Y. 1943), 2018. 155(6): p. 1971-1984.e4. 
51. Yu, Y., et al., STING-mediated inflammation in Kupffer cells contributes to progression of nonalcoholic steatohepatitis. The Journal of Clinical Investigation, 2019. 129(2): p. 546-555.

\section{Tables}

Table 1. Nutritional composition of the experimental NASH diet and standard chow

\begin{tabular}{|lll|}
\hline Dietary Composition & $\begin{array}{l}\text { Standard } \\
\text { Chow }\end{array}$ & Fast food diet \\
\hline Total fat (\% weight) & $4.8 \%$ & $21 \%$ \\
\hline Saturated & $0.93 \%$ & $14 \%$ \\
\hline Mono-unsaturated & $0.99 \%$ & $6.23 \%$ \\
\hline Polyunsaturated & $2.20 \%$ & $0.77 \%$ \\
\hline Cholesterol (\% weight) & $0 \%$ & $2 \%$ \\
\hline $\begin{array}{l}\text { Carbohydrates (\% } \\
\text { weight) }\end{array}$ & $59.4 \%$ & $49.9 \%$ \\
\hline Protein (\% weight) & $20 \%$ & $21 \%$ \\
\hline Fibre (\% weight) & $4.7 \%$ & $\begin{array}{l}4.7 \% \\
\text { Drinking Water }\end{array}$ \\
\hline
\end{tabular}

Table 2. Immunohistochemistry and Immunofluorescence antigen retrieval, primary and secondary antibodies 


\begin{tabular}{|c|c|c|}
\hline $\begin{array}{l}\text { Antigen } \\
\text { Retrieval }\end{array}$ & Primary Antibody & Secondary Antibody \\
\hline \multirow{6}{*}{$\begin{array}{l}10 \mathrm{mM} \\
\text { Sodium } \\
\text { Citrate }(\mathrm{pH} 6)\end{array}$} & $\begin{array}{l}\text { Wide spectrum screening } \\
\text { cytokeratin (Pan-CK) }\end{array}$ & \multirow[t]{2}{*}{$\begin{array}{l}\text { Biotinylated Goat Anti-Rabbit IgG (BA-100, Vector } \\
\text { Laboratories, Meadowbrook, QLD, Australia, 1:150) }\end{array}$} \\
\hline & $\begin{array}{l}\text { (Z0622, Dako, Mulgrave, } \\
\text { Victoria, Australia, 1:200) }\end{array}$ & \\
\hline & $\begin{array}{l}\text { Rabbit Myeloperoxidase } \\
\text { (MPO) }\end{array}$ & \multirow{2}{*}{$\begin{array}{l}\text { Donkey anti-rabbit } 568 \\
\text { (Alexa Fluor conjugates, Life Technologies, } \\
\text { Frederick, MD, United States, 1:500) }\end{array}$} \\
\hline & $\begin{array}{l}\text { (ab45977, Abcam, } \\
\text { Cambridge, MA, United } \\
\text { States, 1:100) }\end{array}$ & \\
\hline & Rabbit CD45 & \multirow{2}{*}{$\begin{array}{l}\text { Goat anti-rabbit } 647 \\
\text { (Alexa Fluor conjugates, Life Technologies, } \\
\text { Frederick, MD, United States, 1:500) }\end{array}$} \\
\hline & $\begin{array}{l}\text { (ab10558, Abcam, } \\
\text { Cambridge, MA, United } \\
\text { States, } 1: 100 \text { ) }\end{array}$ & \\
\hline \multirow[t]{4}{*}{$\begin{array}{l}\text { Tris-EDTA } \\
\text { Buffer (pH 9) }\end{array}$} & $\begin{array}{l}\text { Rat Anti-Neutrophil (NIMP- } \\
\text { R14) }\end{array}$ & \multirow{2}{*}{$\begin{array}{l}\text { Goat anti-rat } 488 \\
\text { (Alexa Fluor conjugates, Life Technologies, } \\
\text { Frederick, MD, United States, 1:100) }\end{array}$} \\
\hline & $\begin{array}{l}\text { (ab2557, Abcam, } \\
\text { Cambridge, MA, United } \\
\text { States, 1:100) }\end{array}$ & \\
\hline & $\begin{array}{l}\text { Rabbit Recombinant Anti- } \\
\text { Heme Oxygenase } 1 \text { (HO-1) }\end{array}$ & \multirow{2}{*}{$\begin{array}{l}\text { Donkey anti-rabbit } 568 \\
\text { (Alexa Fluor conjugates, Life Technologies, } \\
\text { Frederick, MD, United States, 1:500) }\end{array}$} \\
\hline & $\begin{array}{l}\text { (ab52947, Abcam, } \\
\text { Cambridge, MA, United } \\
\text { States, 1:100) }\end{array}$ & \\
\hline
\end{tabular}

Table 3. Real-time Quantitative PCR Primers 


\begin{tabular}{|c|c|c|}
\hline & PCR Primers & Sequence \\
\hline \multirow[t]{24}{*}{ MOUSE } & $m$ Nox2-FWD & TGT CAT TCT GGT GTG GTT GG \\
\hline & $m$ Nox2-REV & GCA GCA GGA TCA GCA TAC AG \\
\hline & $m$ Nox4-FWD & CCA GAA TGA GGA TCC CAG AA \\
\hline & mNox4-REV & ACC ACC TGA AAC ATG CAA CA \\
\hline & mSting-FWD & CTA CAT TGG GTA CTT GCG GTT \\
\hline & mSting-REV & GCA CCA CTG AGC ATG TTG TTA TG \\
\hline & $m / l-6-F W D$ & ATG GAT GCT ACC AAA CTG GAT \\
\hline & $m I l-6-R E V$ & TGA AGG ACT CTG GCT TTG TCT \\
\hline & mTweak-FWD & TTG GCC TCC TGC TGG TCG TGG TCA \\
\hline & mTweak-REV & CTC CCG GCG GTC CTC TGC TGT CA \\
\hline & $m / f n y-F W D$ & GCG TCA TTG AAT CAC ACC TG \\
\hline & $m l f n \gamma$-REV & TGA GCT CAT TGA ATG CTT GG \\
\hline & $m R n 18 s-F W D$ & GTA ACC CGT TGA ACC CCA TT \\
\hline & $m R n 18 s-R E V$ & CCA TCC AAT CGG TAG TAG CG \\
\hline & mIfit1-RT-FWD & GAG AGT CAA GGC AGG TTT CT \\
\hline & mIfit1-RT-REV & TCT CAC TTC CAA ATC AGG TAT GT \\
\hline & $m / f n \beta 1-F W D$ & CCC TAT GGA GAT GAC GGA GA \\
\hline & mIfn $\beta 1$-REV & CCC AGT GCT GGA GAA ATT GT \\
\hline & mRsad2-FWD & CTG TGC GCT GGA AGG TTT \\
\hline & mRsad2-REV & ATT CAG GCA CCA AAC AGG AC \\
\hline & m/sg15-FWD & CAA TGG CCT GGG ACC TAA AG \\
\hline & m/sg15-REV & TAA GAC CGT CCT GGA GCA CT \\
\hline & mlfih 1-FWD & TCT TGG ACA CTT GCT TCG AG \\
\hline & mlfih 1-REV & TCC TTC TGC ACA ATC CTT CTC \\
\hline
\end{tabular}

\section{Figures}




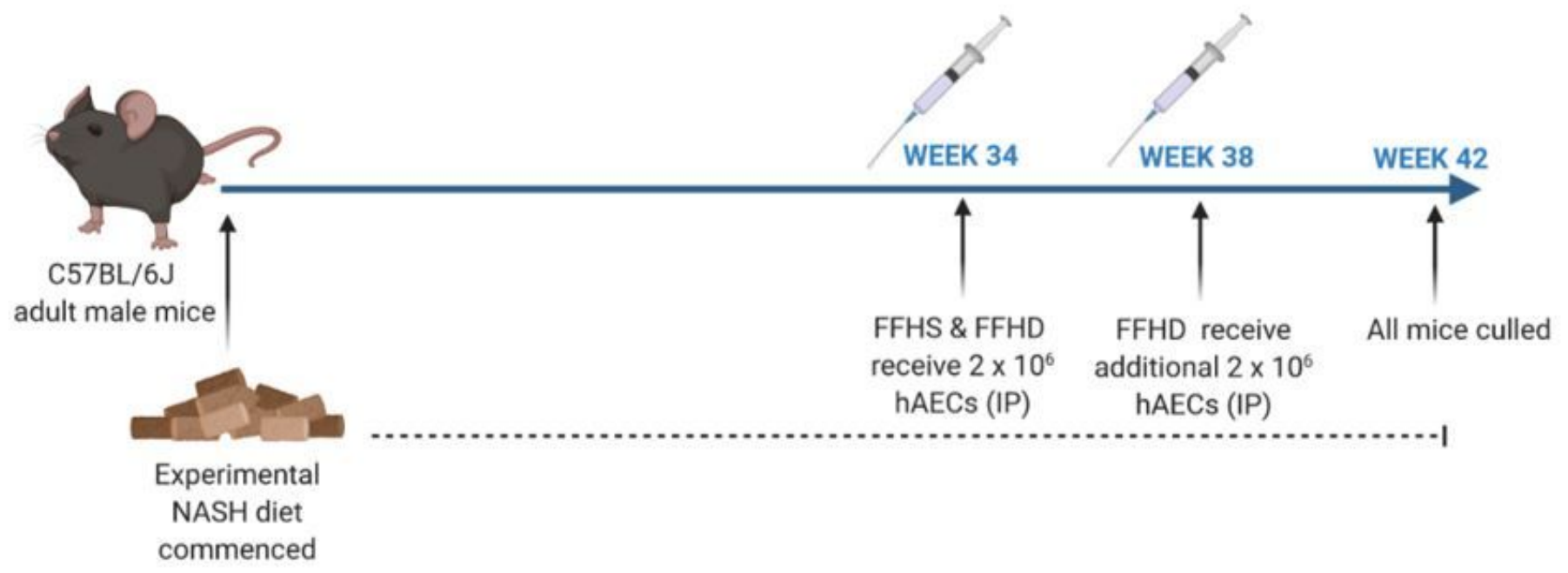

Figure 1

Schedule for experimental NASH diet and hAEC administration Experimental NASH was induced in C57BL/6J male mice using a high-fat, high fructose diet for 42 weeks. Mice received either a single intraperitoneal injection of $2 \times 106$ hAECs at week 34 or an additional hAEC dose at week 38 .
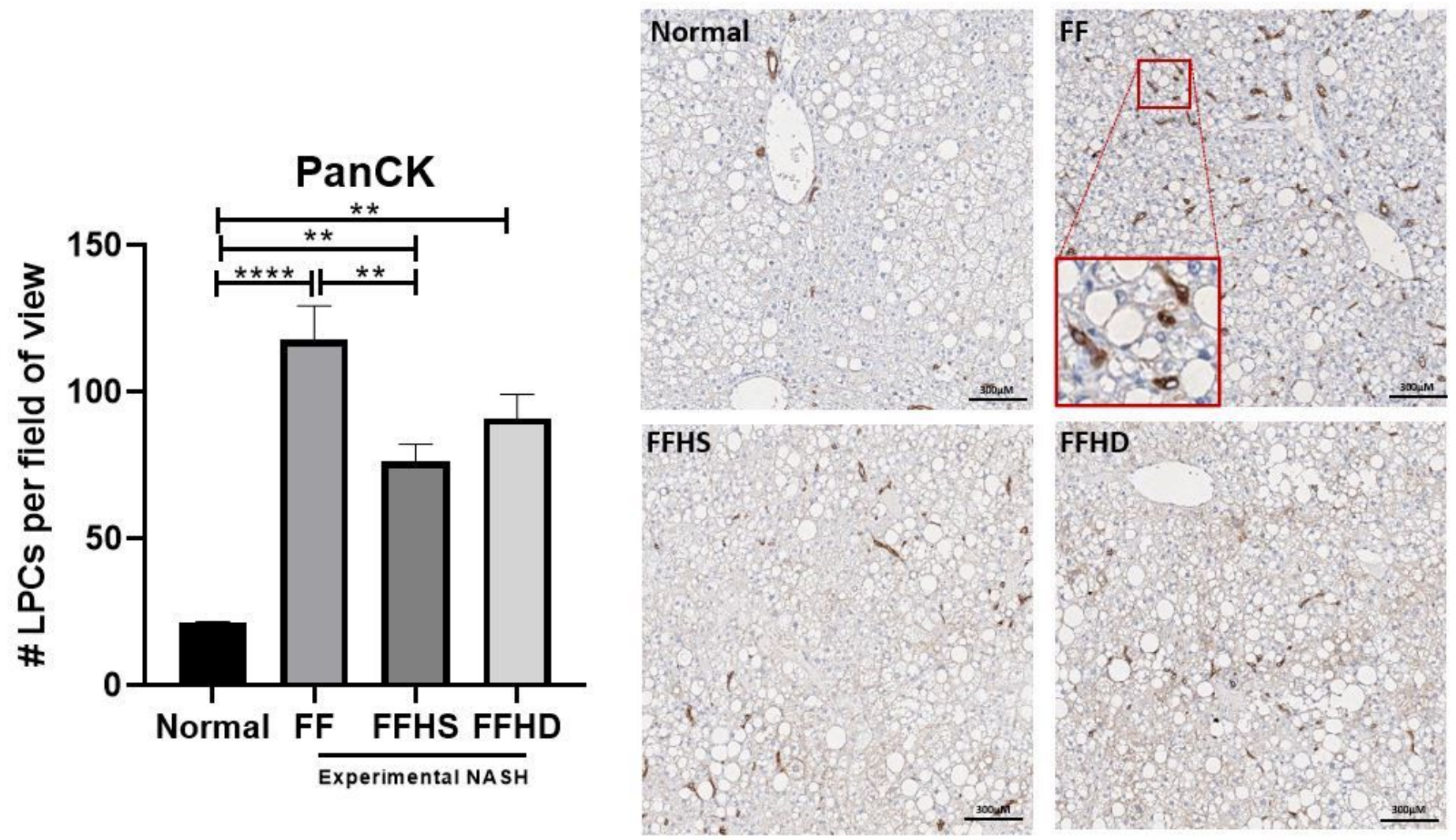

Figure 2 
The effect of hAECs on liver progenitor cell numbers All mice on the NASH diet, (FF, FFHS and FFHD) had a significantly greater number of liver progenitor cells (LPC) compared with normal mice. Within the NASH diet groups, mice treated with a single dose of hAECs (FFHS) had significantly fewer LPCs than FF mice. Magnification: 10x, scale bar $=300 \mu \mathrm{m} .{ }^{\star *} \mathrm{P}<0.01,{ }^{\star \star \star \star *} \mathrm{P}<0.0001$. FF, NASH diet alone; FFHD, NASH diet with double hAEC dose; FFHS, NASH diet with single hAEC dose; hAECs, human amnion epithelial cells; LPC, liver progenitor cell.
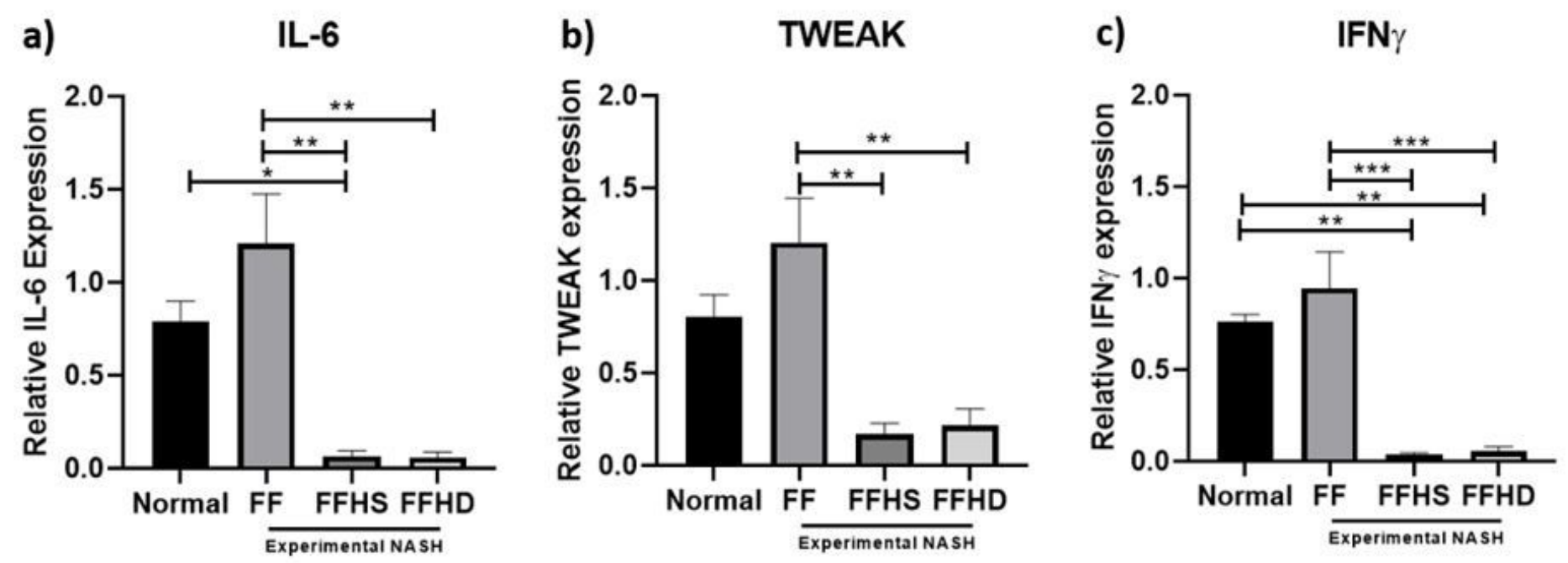

Figure 3

The effect of hAECs on liver progenitor cell mitogens, IL-6, TWEAK and IFNY. Compared with FF mice, FFHS and FFHD mice had significantly lower expression levels of II-6 (a), Tweak (b), and Ifny (c). Compared with normal mice, II-6 gene expression was significantly lower in FFHS mice (a) and Ifny gene expression was significantly lower in mice treated with both a single and double dose of hAECs (c). *P < $0.05, * \star \mathrm{P}<0.01,{ }^{\star \star *} \mathrm{P}<0.001$. FF, NASH diet alone; FFHD, NASH diet with double hAEC dose; FFHS, NASH diet with single hAEC dose. 

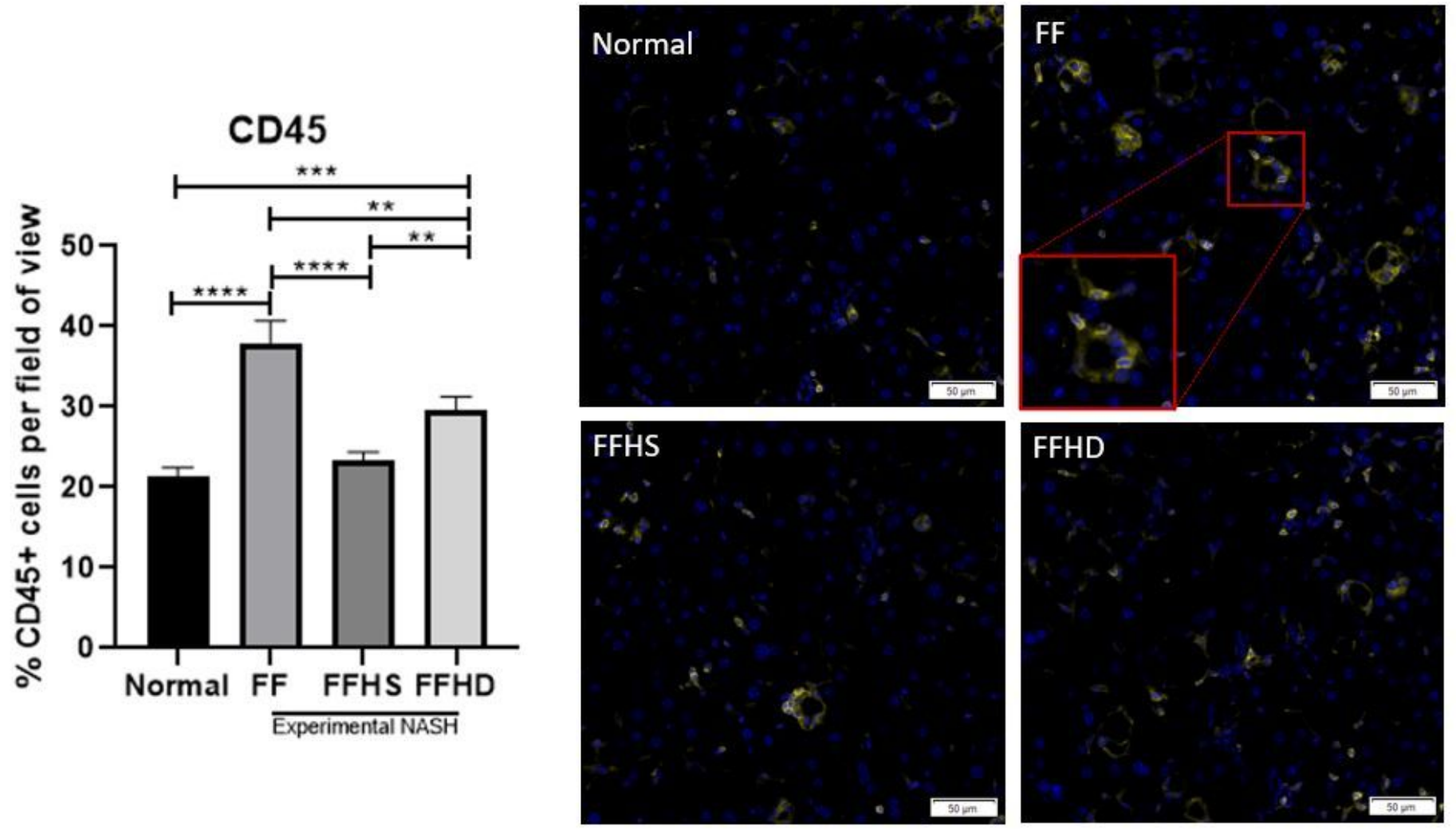

Figure 4

hAECs reduce levels of hepatic leukocytes Compared with normal mice, mice on the NASH diet only (FF) and mice treated with a double dose of hAECs (FFHD) had significantly more hepatic leukocytes (CD45+ cells). Within the NASH diet groups, both FFHS and FFHD mice had significantly lower levels of hepatic leukocytes compared to untreated FF mice. Within the mice treated with hAECs, FFHS mice had significantly fewer hepatic leukocytes compared to FFHD mice. Magnification: 10x, scale bar $=50 \mu \mathrm{m}$. $\star \star P<0.01, * \star \star P P 0.001, * \star \star * P<0.0001$. 

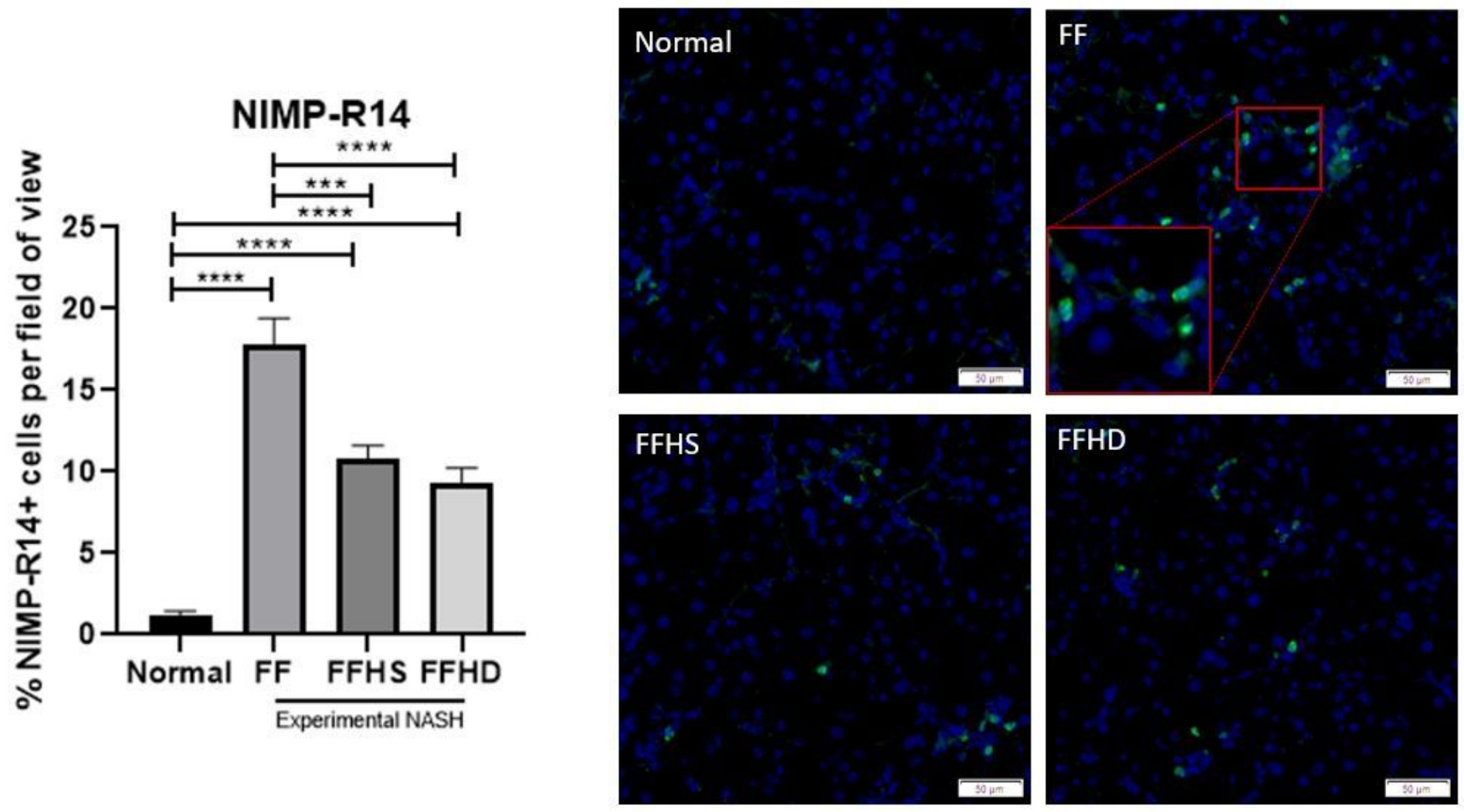

\section{Figure 5}

The effect of hAECs on neutrophil infiltration Compared with normal mice, mice on the NASH diet, had significantly more neutrophils (NIMP-R14+ cells). Within the NASH diet groups, FFHS and FFHD mice had significantly lower levels of neutrophils compared to untreated FF mice. Magnification: 10x, scale bar $=$ $50 \mu \mathrm{m} .{ }^{* \star *} \mathrm{P}<0.001,{ }^{\star \star \star *} \mathrm{P}<0.0001$. 

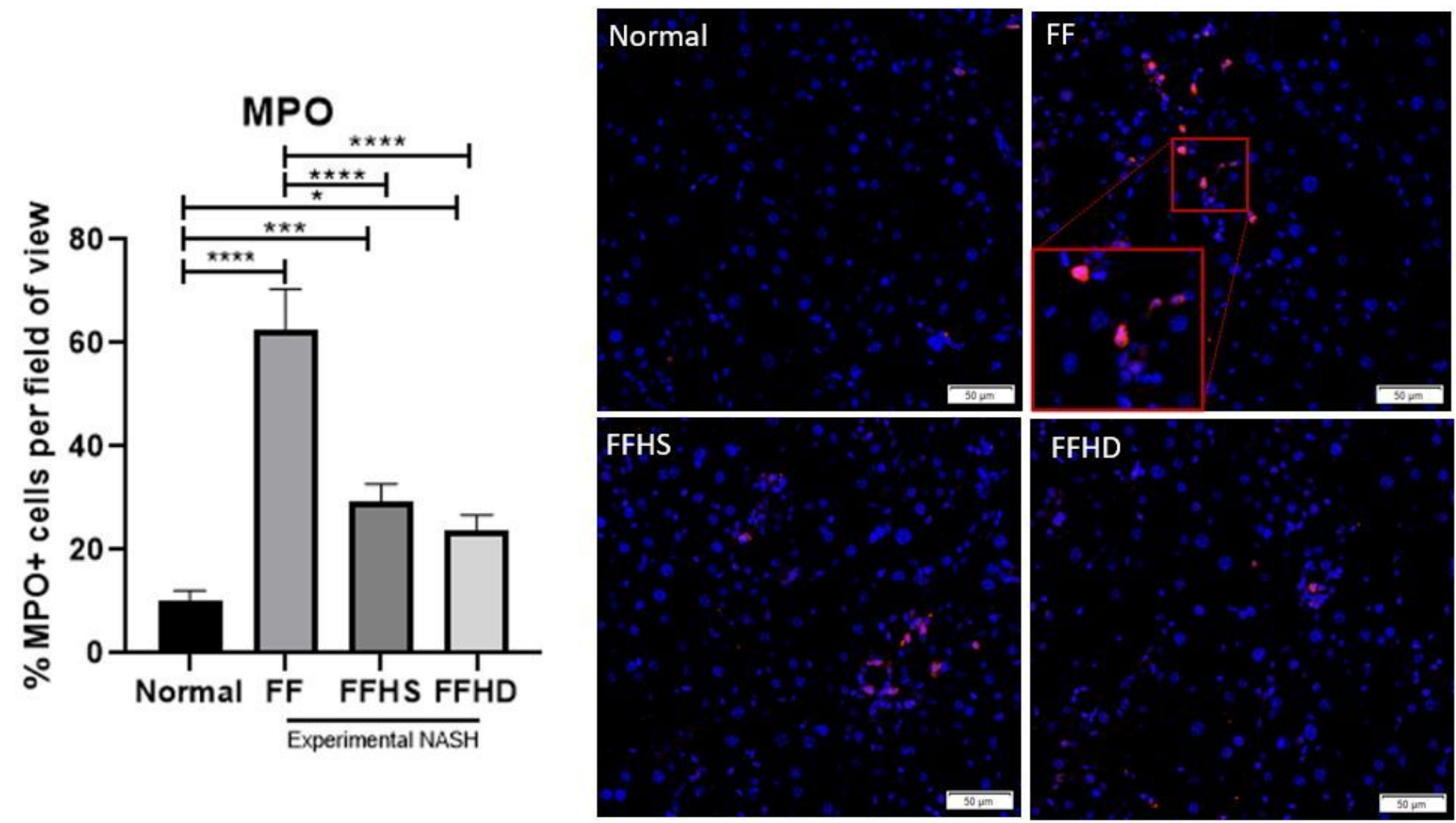

\section{Figure 6}

hAECs reduce myeloperoxidase production Compared with normal mice, mice on the NASH diet, (FF, FFHS and FFHD) had significantly greater levels of MPO activity (MPO+ cells). Within the NASH diet groups, FFHS and FFHD mice had significantly lower levels of MPO activity compared to untreated FF mice. Magnification: 10x, scale bar $=50 \mu \mathrm{m} .{ }^{\star * \star} \mathrm{P}<0.001,{ }^{\star} * \star * \mathrm{P}<0.0001$. MPO, myeloperoxidase. 
a)

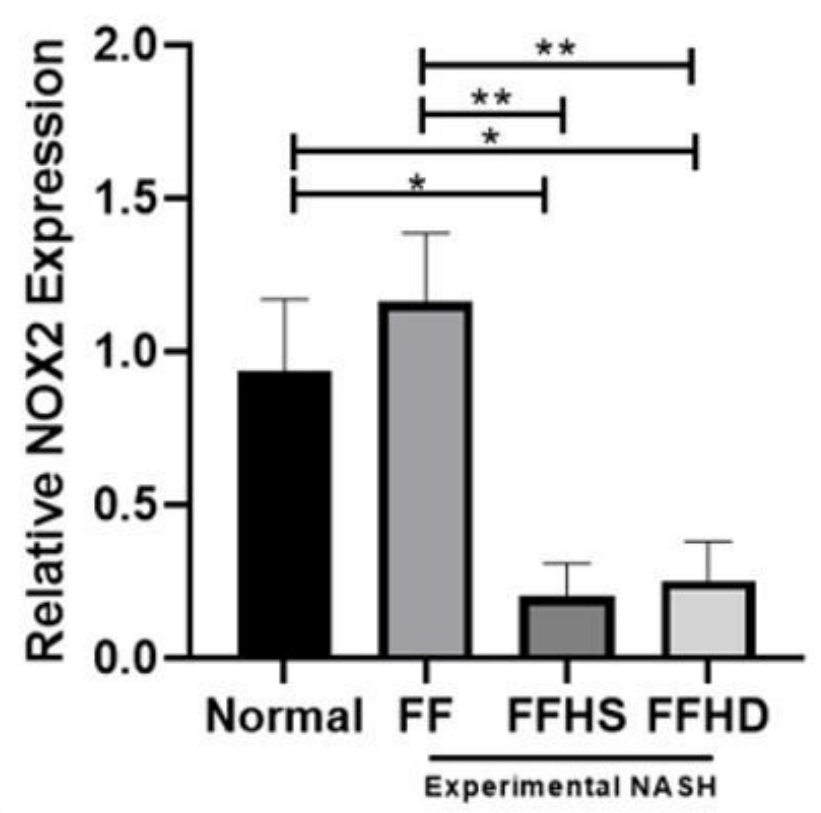

b) NOX4

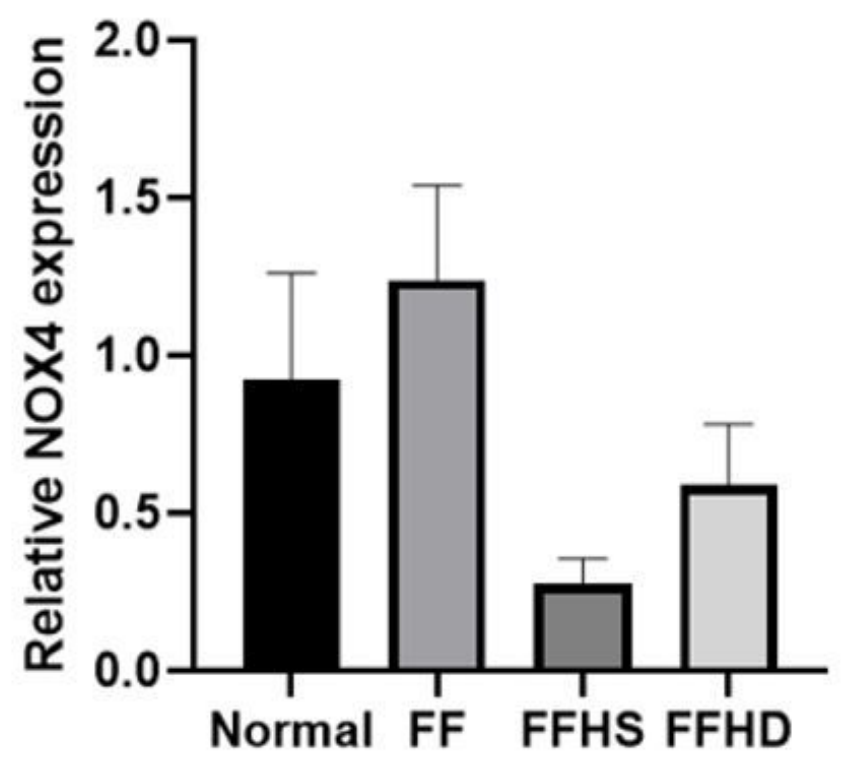

Experimental NASH

Figure 7

Antioxidant effects of hAECs in experimental NASH Compared with normal mice, FFHS and FFHD mice had significantly lower expression levels of Nox2 (a). Within the NASH diet groups, FFHS mice had significantly lower expression levels of Nox2 (a). Nox4 gene expression demonstrated a trend toward lower levels in hAEC exposed mice but did not reach statistical significance $(P=0.10)$. ${ }^{*}<0.05$, ${ }^{\star *} P<$ 0.01 . 

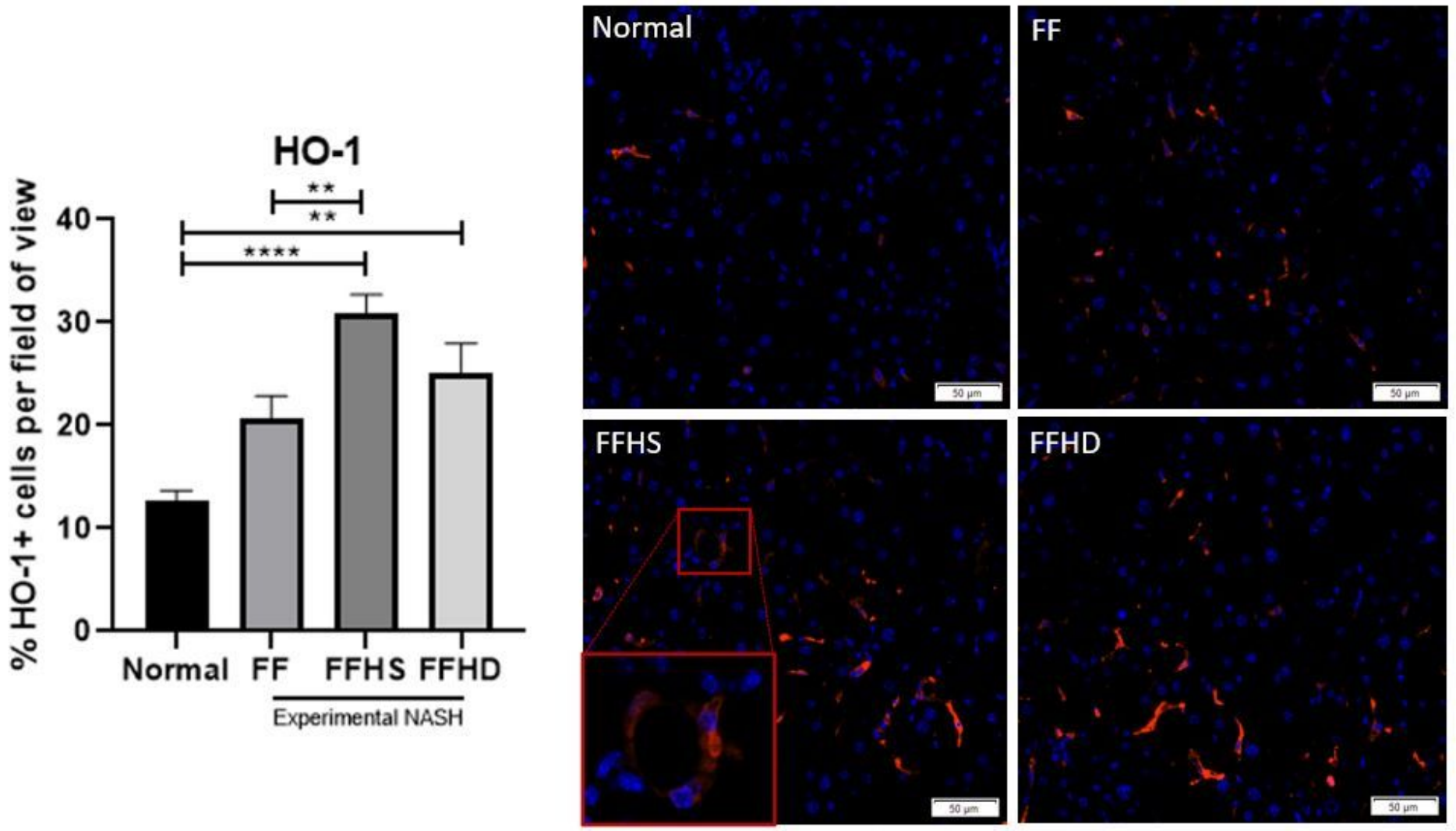

Figure 8

hAECs increase Heme Oxygenase-1 production Compared with normal mice, FFHS and FFHD mice had significantly greater levels of HO-1 activity (HO-1+ cells). FFHS mice had significantly higher levels of HO1 activity compared to untreated FF mice. Magnification: 10x, scale bar $=50 \mu \mathrm{m} .{ }^{\star *} \mathrm{P}<0.01$, ${ }^{\star \star \star \star} \mathrm{P}<$ 0.0001. HO-1, Heme-oxygenase. 

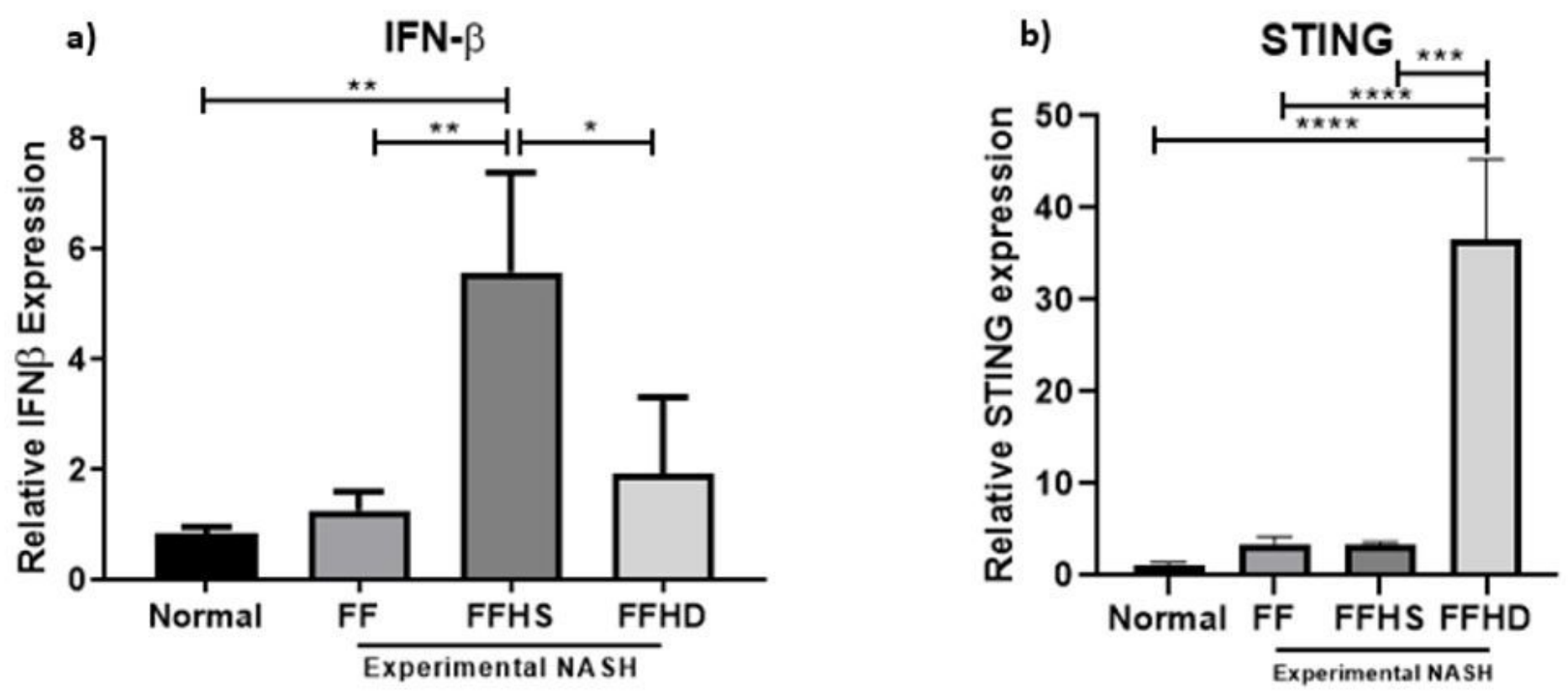

Figure 9

The effect of hAECs on hepatic IFN- $\beta$ and STING levels Compared with normal mice, FFHS mice had significantly higher expression levels of IFN- $\beta$ (a). Within the experimental NASH diet groups, only FFHS mice had significantly higher expression levels of Ifn- $\beta$ (a). The Ifn- $\beta$ expression levels were significantly higher in FFHS mice compared to FFHD mice. Compared with normal mice, FF, FFHS and FFHD mice had significantly higher expression levels of Sting (b). ${ }^{\star} P<0.05, * \star P<0.01, * \star P<0.001,{ }^{\star * \star \star} P<0.0001$.
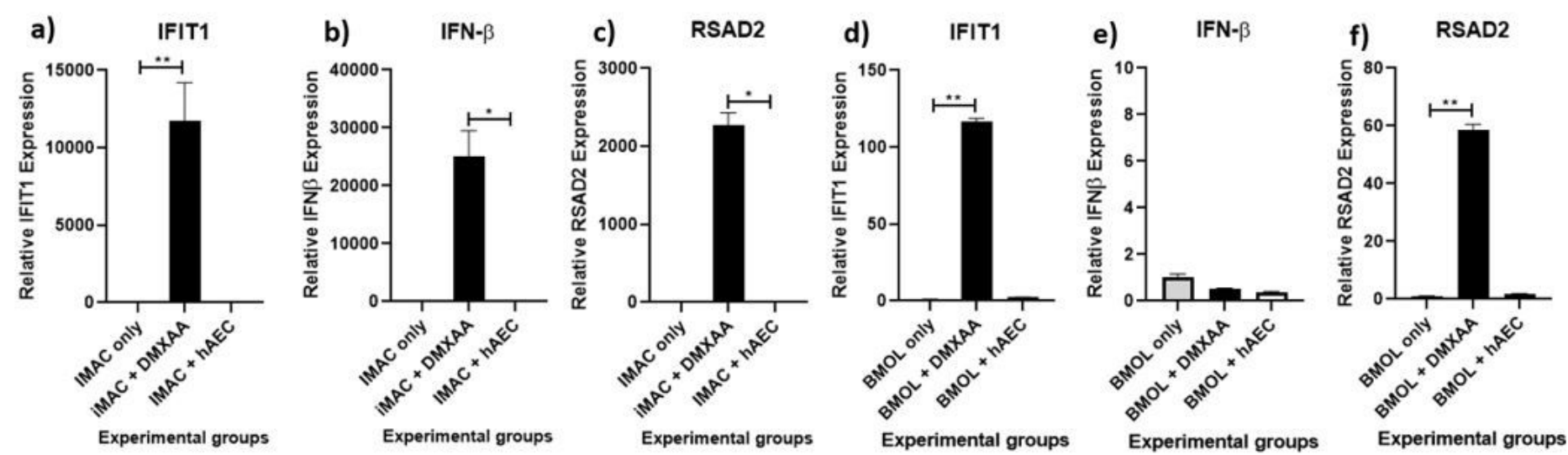

Figure 10

The effect of hAECs on the c-GAS-STING pathway cGAS-STING activation was measured through Ifit1, Ifn- $\beta$ and Rsad2 gene expression in iMACs and BMOLs co-cultured with hAECs. 5,6-Dimethylxanthenone4-acetic Acid (DMXAA) treated iMACs and BMOLs served as positive controls. Compared to untreated IMACs, there was no significant difference in Ifit1 (a), Ifn- $\beta$ (b) and Rsad2(c) in hAEC treated iMACs. 
Compared to untreated BMOLs, there was no significant difference in Ifit1 (d), Ifn- $\beta$ (e) and Rsad2(f) in hAEC treated BMOLs. ${ }^{*}<<0.05,{ }^{\star} \mathrm{P}<0.01$. iMACs, immortalised macrophages; BMOLS, Bipotential Murine Oval Liver cells. 